\title{
Chapitre III - L'histoire des entreprises
}

\section{Maurice Wolkowitsch}

\section{(2) OpenEdition}

\section{Journals}

Édition électronique

URL : https://journals.openedition.org/rhcf/1311

DOI : 10.4000/rhcf.1311

Éditeur

Rails \& histoire

Édition imprimée

Date de publication : 10 février 2004

Pagination : 68-89

ISBN : 0996-9403

ISSN : 0996-9403

Référence électronique

Maurice Wolkowitsch, "Chapitre III - L'histoire des entreprises », Revue d'histoire des chemins de fer [En ligne], 30 | 2004, mis en ligne le 27 août 2012, consulté le 22 avril 2022. URL : http:// journals.openedition.org/rhcf/1311; DOI : https://doi.org/10.4000/rhcf.1311 


\section{Chapitre III \\ L'histoire des entreprises}

'histoire d'une entreprise commence par une candidature
posée pour obtenir la concession d'une ou de plusieurs lignes dans le but de les construire et de les exploiter ${ }^{1}$. Chaque concession a sa propre histoire. En examinant pour chaque ligne ou chaque réseau la liste des concessionnaires qui se sont succédé, on accède à l'histoire des entreprises à travers celles des concessions. Pour une entreprise à réseaux multiples, le regroupement de la documentation permet de la connaitre, d'apprécier par exemple sa politique d'expansion, comme le montre le cas de la SGCFE en Gironde, exposé plus loin.

\section{Trois départements en exemple}

\section{. Département de la Haute-Saône (tabl. 1) ${ }^{2}$}

Ce département comprend, au Nord-Est, une faible étendue des Vosges ; les plaines et surtout les plateaux, plus élevés au Nord, occupent une place majeure. À côté d'une agriculture médiocre, une activité industrielle prospère se développe, dispersée dans des ateliers (petite métallurgie, travail du bois) ou à domicile (textile) ; cette économie alimente une demande de transport soutenue à l'époque du développement des réseaux secondaires ; le réseau d'intérêt général dessert incomplètement ce département. Les conditions sont favorables au développement de lignes secondaires.

La Compagnie générale des chemins de fer vicinaux accepte la rétrocession de la première ligne construite entre Gray et Gy, à la condition d'avoir la préférence pour toute extension du réseau départemental, sauf si des conditions plus avantageuses que les siennes étaient soumises au conseil général. Le réseau atteindra $466 \mathrm{~km}$, plus quelques prolongements dans les départements limitrophes. Le choix de la voie métrique, comme sur la ligne initiale, permet une construction et une exploitation

1- Voir en annexe l'avant-projet proposé pour la section Hyères - Saint-Raphäl de la ligne de Toulon à Fréjus - Saint-Raphaël (annexe 1, p. 77)

2- Les tableaux 1, 2 et 3 ont été établis en utilisant largement l'Encyclopédie générale des transports. Chemins de fer, Valignat, Éditions de l'Ormet, 1990, Hérault, 20 p., Gironde, 26 p., également les articles cités, notamment pour la Haute-Saône, enfin sur des recherches personnelles. Les documents de l'Ormet comportent des cartes précises et des tableaux des ouvertures et fermetures de lignes. 
économiques. La compagnie exerce un monopole, seulement écorné par $3 \mathrm{~km}$ de ligne de la Régie départementale des chemins de fer de la Côted'Or. L'histoire de la compagnie se confond avec celle des chemins de fer secondaires, qu'elle est seule à exploiter dans le département ${ }^{3}$.

\section{Tableau 1. Le département de la Haute-Saône}

\begin{tabular}{|c|c|}
\hline 1872 & $\begin{array}{l}\text { Demande de concession de la ligne de Gray à G } \\
\text { Burdin et Cornu }\end{array}$ \\
\hline 1872 & Avis favorable de la Commission départementale \\
\hline 1873 & Concession accordée à MM. Martin, Pradines fré \\
\hline 1874 & DUP en vue de la construction d'un CFIL \\
\hline 1877 & $\begin{array}{l}\text { Création de la Compagnie anonyme du chemin d } \\
\text { et prolongements }\end{array}$ \\
\hline 1878 & Mise en service \\
\hline 1885 & Liquidation de la compagnie \\
\hline 1886 & $\begin{array}{l}\text { Mise sous séquestre, exploitation confiée à l'adr } \\
\text { Ponts et Chaussées. Le département devenu pr } \\
\text { et du matériel cherche un nouveau concessionn }\end{array}$ \\
\hline 1887 & Concession à la Compagnie des chemins de fer \\
\hline 1888 & Rétrocession à la Compagnie générale des cher \\
\hline 1937 & $\begin{array}{l}\text { Exploitation de nombreuses lignes par autobus } \epsilon \\
\text { progressive du service des marchandises }\end{array}$ \\
\hline \multirow{2}{*}{1938} & Réseau ferré limité à 147 km sur 467 construits \\
\hline & Le conseil général fixe au 30 juin l'arrêt de l'expl \\
\hline 1938 & Le déferrage des voies est entrepris \\
\hline
\end{tabular}

\section{. Département de l'Hérault (tabl. 2)}

Le relief oppose la plaine du Languedoc, relativement étroite, à la bordure du Massif central de pénétration difficile. La viticulture domine dans la plaine, elle subit la crise du phylloxéra et ses séquelles : la production a diminué de moitié, or cette activité alimentait une grande partie du trafic des marchandises. Dans les premiers contreforts de la montagne et la montagne elle-même, hors les dépressions, une agriculture archaique ne peut fournir beaucoup d'échanges. L'industrie textile faiblement représentée est localisée à Ganges desservie par le PLM par une ligne au tracé difficile.

3- J. Finsterwald et C. Bouchaud, «Les chemins de fer vicinaux de la Haute-Saône de l'extension (1878-1917) au déclin (1918-1938) », Chemins de fer régionaux et urbains, $\mathrm{n}^{\circ} 287\left(2001, \mathrm{n}^{\circ}\right.$ 5) p. 4-15. 
Comme en Haute-Saône, la Compagnie des chemins de fer de l'Hérault exerce un monopole, à peine contrarié par un prolongement de $20 \mathrm{~km}$ d'une ligne exploitée par la Compagnie des tramways à vapeur de l'Aude. La similitude s'arrête là : le réseau ferré total de l'Hérault est de $643 \mathrm{~km}$ dont $63 \%$ de lignes d'intérêt général, en Haute-Saône la proportion est de $904 \mathrm{~km}$ et $48 \%$. Midi et PLM se rejoignent à Sète ; leurs lignes sont concentrées dans la plaine ; les lignes secondaires, imbriquées dans les réseaux d'intérêt général, ont de multiples points de jonction avec eux, si bien que le trafic leur échappe ; les parcours des voyageurs et des marchandises sont brefs. Si l'on ajoute à cela le choix de l'écartement normal, avec le coût qu'il entraine, on conçoit que les conditions sont peu favorables au développement des lignes secondaires. Possédant des villes importantes, l'Hérault a plusieurs réseaux de tramways : l'histoire de ceux de Montpellier et Béziers a retenu notre attention ; celui de Sète a une histoire parallèle et a fonctionné peu de temps, nous ne l'avons pas étudié.

Tableau 2. Le département de l'Hérault

\begin{tabular}{|c|l}
\hline $\mathbf{1}$ - Com pagnie des CFL de l'Hérault \\
\hline 1866 & Demande de concession de M. Joret \\
\hline 1867 & DUP accordée pour 5 lignes \\
\hline 1872 & Mise en exploitation de la ligne Montpellier-Pa \\
\hline $1873-1878$ & Plusieurs mises en exploitation \\
\hline 1879 & Difficultés financières \\
\hline 1884 & Faillite à titre conservatoire \\
\hline $1887-1897$ & $\begin{array}{l}\text { Achèvement du réseau ; dernière mise en s } \epsilon \\
\text { Marseillan }\end{array}$ \\
\hline 1928 & Le département rachète la compagnie et l'aff \\
\hline 1932 & $\begin{array}{l}\text { Le conseil général décide la fermeture du rés } \\
\text { maintien de la ligne Montpellier - Palavas four } \\
\text { trafic voyageurs }\end{array}$ \\
\hline 1939 & $\begin{array}{l}\text { Rétablissement du trafic sur toutes les lignes } \\
\text { irrégulières }\end{array}$ \\
\hline $1947-1968$ & Arrêt progressif de l'exploitation \\
\hline $\mathbf{2 - T r a m ~ w a y s ~ d e ~ M o n t p e l l i e r ~}$ \\
\hline 1875 & $\begin{array}{l}\text { Demande de concession de M. Francq pour } \\
\text { divisée en } 6 \text { lignes de TV hippomobile }\end{array}$ \\
\hline 1877 & DUP \\
\hline 1879 & Rétrocession à la Compagnie générale des o \\
\hline $1880-1881$ & Achèvement du réseau \\
\hline 1882 & Faillite. Le syndic poursuit l'exploitation \\
\hline
\end{tabular}


(Suite du tableau 2)

\begin{tabular}{|cl}
\hline \multicolumn{2}{|c}{ Tramways de Montpellier (suite) } \\
\hline Fin 1882 & $\begin{array}{l}\text { Exploitation suspendue. Dépose des voies } \mathrm{p} \text { c } \\
\text { Chaussées }\end{array}$ \\
\hline 1891 & Déchéance de la compagnie \\
\hline 1894 & Projet d'un réseau de tramw ays électriques \\
\hline 1897 & Concession pour 5 lignes à voie métrique \\
\hline 1898 & Mise en exploitation des 5 lignes \\
\hline 1920 & Fermeture de 2 lignes \\
\hline 1947 & Expiration de la concession. Exploitation en r \\
\hline 1948 & Les tramw ays cessent de circuler \\
\hline
\end{tabular}

\begin{tabular}{|c|l}
\hline \multicolumn{2}{|l}{ 3 - Tram ways de Béziers } \\
\hline 1878 & DUP pour un TV à vapeur reliant Béziers à V \\
\hline 1879 & Mise en service \\
\hline 1880 & Rétrocession \\
\hline 1882 & Remplacement de la traction à vapeur par la 1 \\
\hline 1885 & Faillite \\
\hline 1886 & $\begin{array}{l}\text { Concession à Messieurs Allignan et Fabre pc } \\
\text { de TV électrique }\end{array}$ \\
\hline $1886-1901$ & $\begin{array}{l}\text { Contrairement à l'engagement, les concessio } \\
\text { traction hippomobile }\end{array}$ \\
\hline 1896 & Le conseil municipal décide le principe du rac \\
\hline 1899 & Concession à la Compagnie des tramw ays d \\
\hline 1901 & $\begin{array}{l}\text { Mise en service des tramw ays électriques SI } \\
\text { lignes }\end{array}$ \\
\hline $1916-1920$ & Fermeture progressive de 3 lignes \\
\hline 1928 & Réseau limité à 2 lignes \\
\hline 1 nnn & Rachat par la ville et déclassement des ligne؟ \\
\hline
\end{tabular}

\section{. Département de la Gironde (tabl. 3)}

Le relief inclut une partie de la plaine des Landes; ailleurs, les collines et les coteaux encadrent les larges vallées de la Garonne et de la Dordogne et bordent l'estuaire ; un plateau occupe l'Entre-Deux-Mers. La production forestière et la viticulture tiennent à l'époque une grande place dans l'économie. Bordeaux, centre de la commercialisation des vins, a aussi une activité portuaire, source de trafics.

Ces conditions favorables ont conduit à la construction d'un réseau de $1019 \mathrm{~km}$ dont 59 \% de lignes d’intérêt général. Les lignes secondaires ont dû s'insinuer entre les lignes des six compagnies en activité dans le département, que l'État voulait protéger contre une trop 
vive concurrence : le Grand-Central en faillite presque avant d'exister ; Médoc et Charentes créées pour répondre à une demande régionale et dont les lignes ont fini dans le giron des grandes compagnies, après un jeu subtil d'échanges et de rachats; PO, Midi et Ouest-État assurant une bonne desserte du département. De multiples compagnies secondaires, dont sept subsistent encore en 1927, construisent un réseau long de $414 \mathrm{~km}$ en 1911. À travers l'histoire de chaque ligne, on suit celle de chaque concession et des entreprises concernées. On est loin des situations de monopole rencontrées en Haute-Saône et dans l'Hérault. Le réseau de TV de Bordeaux a une histoire simple, étant soumis seulement à deux gestionnaires en 60 ans avant d'être placé en régie.

L'histoire des entreprises est encadrée par des procédures imposées et soumise à des règles strictes. Cependant, l'histoire des entreprises observée à travers leur comportement dans le cadre des concessions auxquelles elles s'intéressaient plus ou moins longtemps montre des schémas d'évolution qu'il convient de définir.

Un autre enseignement de ces analyses est que chaque département est un cas, méritant une étude minutieuse des divers facteurs pesant sur les choix en matière de construction de chemins de fer secondaires.

Tableau 3. Le département de la Gironde

\begin{tabular}{|c|c|}
\hline \multicolumn{2}{|c|}{1 - La Compagnie de chemin de fer du Médoc } \\
\hline 1857 & Concession d'une ligne Bordeaux-Le Verdon \\
\hline 1861 & Déchéance \\
\hline 1863 & $\begin{array}{l}\text { Concession pour une ligne d'intérêt général à } \\
\text { chemin de fer du Médoc fondée par des nota } \\
\text { ligne sera ouverte en huit étapes (1868-1875 }\end{array}$ \\
\hline 1878 & $\begin{array}{l}\text { Concession à la Compagnie de la ligne d'intér } \\
\text { Castelnau }\end{array}$ \\
\hline 1884 & Mise en service de la liaison Margaux-Castell \\
\hline 1884 & $\begin{array}{l}\text { Concession de } 500 \mathrm{~m} \text { de ligne d'intérêt local } \\
\text { des-Pilotes }\end{array}$ \\
\hline 1888 & Mise en service de la liaison Pauillac - Port-d \\
\hline 1908 & Concession d'une ligne d'intérêt local Casteln \\
\hline 1910 & Le Midi rachète la Compagnie du Médoc \\
\hline 1911 & $\begin{array}{l}\text { Le Midi rétrocède à la Compagnie de chemin } \\
\text { Marensin, filiale de la Compagnie du Médoc, la } \\
\text { Margaux - Sainte-Hélène }\end{array}$ \\
\hline 1915 & Ouverture de la ligne Margaux - Sainte-Hélèr \\
\hline 1917 & $\begin{array}{l}\text { Cession de la ligne Margaux - Sainte-Hélène } \\
\text { Born et Marensin à la Compagnie des voies } f\end{array}$ \\
\hline
\end{tabular}


(Suite du tableau 3)

\begin{tabular}{|c|c|}
\hline \multicolumn{2}{|c|}{2 - Ligne de I'Entre-Deux-Mers : Bordeaux - La Sauve } \\
\hline 1860 & Premier projet refusé par le conseil général \\
\hline 1866 & Projet présenté par MM. Riché et Chrétien \\
\hline 1869 & Concession à MM. Riché et Chrétien \\
\hline 1869 & DUP \\
\hline 1873 & Mise en service \\
\hline 1874 & Rachat par la Compagnie des Charentes \\
\hline 1878 & Rachat par l'Ouest, fin du régime d'intérêt loc \\
\hline 1883 & Intégration au PO, prolongement jusqu'à Eymı \\
\hline \multicolumn{2}{|c|}{3 - Ligne des Landes (concessions d'origine et rach } \\
\hline 1869 & $\begin{array}{l}\text { Concession à MM. Faugère et Bernard d'une } \\
\text { Saint-Symphorien }\end{array}$ \\
\hline 1870 & DUP \\
\hline 1873 & Mise en service \\
\hline 1876 & Ouverture de la section Saint-Symphorien à? \\
\hline 1876 & $\begin{array}{l}3 \text { conventions et } 1 \text { avenant pour autoriser le } \\
\text { Luxey (Landes) de la ligne de Nizan à Sore }\end{array}$ \\
\hline 1885 & Loi entérinant les procédures de 1876 \\
\hline 1886 & Mise en service de la liaison Sore-Luxey \\
\hline 1886 & Convention prévoyant le rachat de la ligne pa \\
\hline 1951 & Fermeture de Nizan - Saint-Symphorien (voy \\
\hline 1954 & Fermeture de Saint-Symphorien à Sore (voye \\
\hline 1971 & Fermeture de Saint-Symphorien à Luxey (ma \\
\hline \multicolumn{2}{|c|}{$\begin{array}{l}4 \text { - Programme d'un réseau de CFIL à voie norm } \\
\text { conseil général }\end{array}$} \\
\hline & Lesparre, Lacanau, Facture, Saint-Symphori \\
\hline
\end{tabular}


(Suite du tableau 3)

\begin{tabular}{|c|c|}
\hline \multicolumn{2}{|l}{ Ligne du Blayais (suite) } \\
\hline 1888 & Mise en service de Blaye à Saint-Ciers-sur-C \\
\hline 1889 & Mise en service de Blaye à Saint-André-de-1 \\
\hline 1954 & Fermeture de la ligne dite du Blayais \\
\hline
\end{tabular}

(a) La Compagnie des chemins de fer économiques des Chá ligne de Saint-Ciers-sur-Gironde à Pons dont $4 \mathrm{~km}$ dans le $\mathrm{d}$ Gironde.

6 - Ligne des Landes et de l'estuaire (b)

Réalisation par la SGCFE du plan établi par le conseil généra

\begin{tabular}{|c|c|}
\hline 1884 & Lesparre, Lacanau, Arès, Facture, Saint-Syr \\
\hline 1885 & $\begin{array}{l}\text { Bordeaux, Bruges, Sainte-Hélène, Lacanau } \epsilon \\
\text { Beautiran }\end{array}$ \\
\hline \multicolumn{2}{|c|}{ Fermetures pour le transport des voyageurs } \\
\hline 1952 & Lesparre, Lacanau, Arès \\
\hline 1954 & $\begin{array}{l}\text { Hostens à Beautiran et Bordeaux à Lacanau } \\
\text { services occasionnels }\end{array}$ \\
\hline 1955 & $\begin{array}{l}\text { Facture, Hostens, Saint-Symphorien ; suppre } \\
\text { occasionnels entre Bordeaux et Lacanau }\end{array}$ \\
\hline 1971 & Arès, Facture \\
\hline \multicolumn{2}{|c|}{$\begin{array}{l}\text { (b) Les dates de fermeture sont plus tardives sur certaines } \\
\text { transport des marchandises. En } 1978 \text {, le trafic est totalemer }\end{array}$} \\
\hline \multicolumn{2}{|c|}{ 7- Ligne de Lacanau à Lacanau-océan } \\
\hline 1886 & Concession à Monsieur Ortal \\
\hline 1889 & DUP \\
\hline 1897 & Exploitation confiée à la SGCFE \\
\hline
\end{tabular}


(Suite du tableau 3)

\begin{tabular}{|c|c|}
\hline \multicolumn{2}{|c|}{ La Teste-Cazaux (suite) } \\
\hline 1886 & Déchéance \\
\hline 1887 & Exploitation en régie directe, puis affermage : \\
\hline 1934 & $\begin{array}{l}\text { Suspension du trafic à l'exception de la dess } \\
\text { Cazaux assurée par l'armée }\end{array}$ \\
\hline \multicolumn{2}{|c|}{10 - Ligne de Bordeaux à Cadillac } \\
\hline 1890 & Projet présenté \\
\hline 1894 & Concession au groupe Faugère \\
\hline 1894 & DUP \\
\hline 1897 & Mise en service \\
\hline 1934 & Rachat \\
\hline 1935 & $\begin{array}{l}\text { Déclassement de la ligne prononcé avant mê } \\
\text { l'exploitation }\end{array}$ \\
\hline \multicolumn{2}{|c|}{11 - Ligne de Saint-André-de-Cubzac à Libourne et $P$} \\
\hline 1908 & Concession pour un TVM à traction électriqur \\
\hline 1909 & DUP \\
\hline 1913 & Mise en service \\
\hline 1949 & Fermeture \\
\hline \multicolumn{2}{|c|}{12 - Tram way d'Arcachon à voie métrique } \\
\hline 1906 & DUP \\
\hline 1906-1910 & Série de concessionnaires qui abandonnent, \\
\hline 1918 & Mise en service \\
\hline 1921 & Mise sous séquestre \\
\hline 1927 & Affermage à Monsieur Ortal \\
\hline 1932 & $\begin{array}{l}\text { Autobus d'une filiale de la Compagnie du Midi } \\
\text { tramw ay, ligne exploitée avec une subventio }\end{array}$ \\
\hline
\end{tabular}

12- Tram in ave No Rnrdasirv 


\section{Les entreprises et leurs concessions}

Les rapports entre concédants et concessionnaires étaient précisés dans des traités ou des conventions énonçant les obligations de chacun. Les difficultés entre les parties étaient fréquentes. Une entreprise incapable de mener à terme la construction ou l'exploitation d'une ligne était vouée à des sorts différents suivant sa situation. Elle pouvait être frappée de déchéance au titre des articles 7 de la loi de 1880 et 30 de la loi de 1913. Au cours de leur vie, les entreprises subissent des modifications de statut, de dénomination, d'extension de leur réseau.

Chaque cas a son histoire ; il est cependant possible de schématiser les étapes qui s'établissent le plus fréquemment entre concédants et concessionnaires.

Pour les CFIL et les TVM, le premier concessionnaire cède la place par abandon ou rétrocession faute de moyens financiers et d'un encadrement technique satisfaisant ; souvent la construction de la ligne n'a pas commencé. Les successeurs construisent la ligne et la mettent en service eux-mêmes ou par le biais d'une société créée à cet effet. Certaines de ces entreprises résisteront jusqu'à l'arrêt de l'exploitation de leur réseau. D’autres seront contrôlées sous divers statuts par les plus grandes entreprises de ce secteur d'activité ou seront rachetées par les concédants soucieux de maintenir la ligne en service ; le rachat intervient surtout à partir de 1920. La concurrence automobile et la crise économique accélèrent cette évolution qui s'achève avec la suspension de l'exploitation par voie ferrée.

Pour les TV, les concessions sont accordées pour des lignes ou des réseaux de 5 à $10 \mathrm{~km}$ parcourus par des tramways à traction hippomobile; les premiers concessionnaires sont souvent capables de les construire et de les exploiter. Les chevaux mal nourris et maltraités sont cause de troubles dans l'exploitation ; aussi, lorsque le tramway électrique a fait ses preuves, presque tous les réseaux en place se convertissent-ils à la nouvelle technique, entre 1890 et 1900, avec appel fréquent à un nouveau concessionnaire. Guerre, concurrence automobile et crise entraînent les mêmes conséquences que pour les réseaux départementaux.

Ces traits d'une évolution commune n'empêchent pas l'existence d'une réelle diversité au sein de ces entreprises, que nous allons nous employer à reconnaître. 


\section{Annexe 1}

Exemple d'un avant projet en vue d'une demande de concession

\section{Département du Var}

CHEMIN DE FER D'INTÉRÊTT LOCAL

LIGNE

DE TOULON A FREJUS-SAINT-RAPHAEL

Tracé par le littoral

Parlie comprise entre Hyeres et StRaphael.

AVANT--PR0JET

\section{MÉMOIRE}

Dressé par l'Ingénieur civil soussigné,

Paris, le

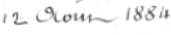

Phésenté par le demandeur en concession soussigné,

Paris, le

13 20u- $1881+$
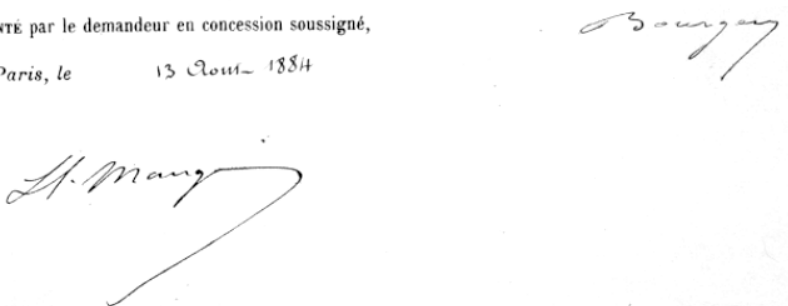


\section{Mémoire justificatif.}

Exposé.

As partí- de Goulon, le chemin de fer-de la Compagnie de Praris à fyon en à la Méditernanée, s'éloigne du littoral, laissan- entre la mer es lui tou le massif montagneux des e rboureno; il decrir entre Goulon es Trejur ungrand are de cercle convexe, quilui perrnes de serapprocherQu chef-lieu du départemeni es de centrer de population importanta, mais il abandonne compléter mem toute la zoner an littozal, arvêté par-ces abandon danr son développemoni, alorr que touter les région bisernalen desservien pras Pa voie ferree, on acquir un accroissemem san. précédent. St suffir d'xilleun O'indiquer, qu'il y a un interer strategique, signalé par les hommer compétentr er un intónes commercial sérieux, à relier par un chemin de fer ler portro ei les rader on monillager de cette partic de la cote. - Les station Givernaler dowem être, pour le départemen bu Yar une sounce de richessers; ler point abriter be rencontrem entre Eoulon en MCyerero, ilr se multuplien jusqu'à Criéjur; le nombre des voyageurs, qui composen 
La clientele spéciale des station d'été en d'hiver, augmente chaque année; he tendance signalée de ces soyageurre, est de sisiter toute ha cote en faisam de courk séjourn dian efaque station; on pa aujound'hin jusqu'en thalies. Or lesplages de favandou, Je la Fossette), de Cavalaine, $\partial_{e}$ ) Sainte. Mtraxime, som aussi sétrisanter, aussi

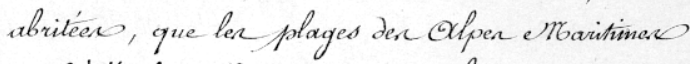
an'bhatie; eller serone aussi fréquentéer $\geqslant$ quand on pourna y arriver facilemen es commodémem. Cu poin de vue du déseloppemenu de l'exploita tion du sol, toin we été dis ovecrim es tou le monde) esh J'accord sur l'ungence de créer des voies nonvellers De communication, danx harégion qui nour öcappe. Le départemen ess done intéressé auplus faur poim, à liétablissemem du chemin de fer, er dès L'année 1881, le Condeil Géréral a prix der Délibérations, pour faire proséder à des études teclyniquess. Son attention d'ess-portée dur touter les Localitén à dessersir en eMb. Mtr. len) Inyenieurs des Prontr en Chausseer om soumin au Conseil, hes étuder des diverc tracéc en présence.

Q V la suito de lienquate ơ laquelle cer avam projetr furene soumin, he Conseil General

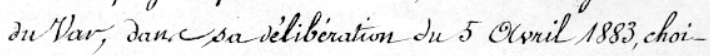
si- definitivemom la direction par le littoral, es érigea, en principe, quil y aurain lieu d'arreter, que Eoulon serain- la tete de ligue 
du tracé. délibiera:

Se \& Septcmbre suivam le Conseil General

1i. Guc la demande de concession d'un chemin de fer à section de 1:", ie Goulon à Tréjure par Hyjeres en to Bropez, en suivam le littoral, étain prise en considération.

2" cou'une fois le projer-résiséer complèké il serain procédé par les soine de ellbonisieun le Préfei on Nar à une enquête administrativel auprèr des mumicipalitén des localités trusersiéero, à lieffer de conmâtre les intentiour den commenerer des proprictáners en la mesure De leurs sacrificenes enfaveur de liexcécution du chomin de fer

(C'est pour aider à la réalisation de ce) programme que le projer actud ess présenté? - Aprés les rapporto si complatro souminas précedenmon an Conseil Général, soir parsen

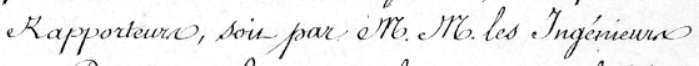
des Pontro a Ofausséer, il paraîn inulite d' in sister, de nousean, sur les avantagen mulipleres au Chemin de for dur littoral entre Foulon et 0 Créjur Saim - Raphael.

Eracé.

Les montagnes des e Mraures projettent vern la mer une série de pointen en de capr, laissam entre enx de waster rudar, dec baien, le golfe de S: Eropen en enfin de magnifiquere plagere?, $q u$ 'une route en construction, ess destince ìs) 
Jesservir J'une facon bien incomplète. Lè tracé du - homin de fer suiu les contowir de la cotes en ne) sien éloigne que pour franchir dann des conditionn, pratiquers d'cxécution, er aussi économiquere que pod-

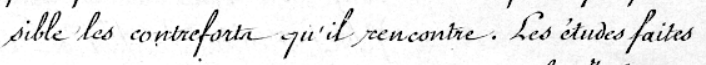

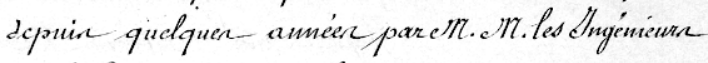
de P'Etar, soir pour les projek de rouk à proxi. mité du littoral, soir pour ler projetr de cheminn de for, on été miser à profin, a le tracé proposé franchin lere passagen difficiler aux points indequés prir les étuderi précédenter er reconnur les souln, possiblen.

COlignemento a. Courbes.

Le' efremin de fer n'étru- aduibsible qu'asece un priar o'exécution modéré, en égard arta difficulién au terricin, t'emploi de courber nombreuser eninduspendable pour contoumer, le longan parcourn, hes nontieuler en les crouper arrondien a littonal. ha tendance Jour être, d'en auguenter le nombre? dauclen ètuder définitiver main le nombre der rapomr acfaible courbure cos, ar restera en faible, proportion, l'exploitution n'aura pasà en souffic. L'examen des plane en du devic dederiptif per met - de s'en rendre compte).

Trofil en long.

$$
\text { Qn trouse danx le profil enlong dere? }
$$
penter es ramper de trente millimetren; on ne pent par les rejeter, car l'objectif à poursuince ess de diminuer la longueur des sonterrainn indispensables, 


\section{5}

quand on n'arrive pas à les supprimer Lens pointr yui nécessitem $\rightarrow$ pour être franchin de fortes rampen, som den pastagen obligen, des colve, oit om abouté néessaircmem- les éluder faites dank la région er oü passem dejä ler router qui desserven - ler villager de la cote). Ces fortes ramper om des inconsémicutic Sanc doute, an poim de vue de liexploitation, mair ces ineonvémientr tenden- à diminuer chacue) jour; grâce aux progrèr imessantre réaliséres Jan la construction du materiel roulam. On a évité soignensemen liemploi combiné des forter, ramper en des courbes à faible ruyon.

Trofilo entrowero. Los profile en travere typer on ététablis suivam les indication fournien parle eafoier Des effarger modele de l' eldnimistration . Leves torlux des trancheer serom inclinér à $45^{\circ}$. dann les forrtien en verre en en schiste décomposé, az $1 / 5$ dane les partien en wocher compoute, es dane les partien en remblai, la pente des talur deroo de 3 de base pour 2 de Gauteur- Oes fossere?, delargeur suffidante, à eause des penter générale. mem-employées, som- prévur dancler profith, pour l'écoulemen des canxy.

Eetradosmento. Les tranchien serom ouverter Jana la terre) végétale sablonneuse danceler schister pluse on minu friablers, dann la, roche compoacte le pluns 


\section{i}

sousem. Les escarpoment Jer coter a des rasine trasersed montrem que les tahlus au 1/5awon en général une bonne tenue), que les glissementrs Dangereux nesom par à redouter, car là où il y a faluise, c'eds que les assidendes strutero som-furorabler à la résistance, ar ailleuro, len penter fortemem adoucien permettrom les tranchés de profondeur tries-atténice?

Les souternain serom-ouvertin dank la roche plus ou moinc aure, schistense a gneissique?; f'cocamen du termain semble montrer que, d'il.y a quelque foin des sonncen superficiellen per. monenters, oucr à liépaisseur dela conche? snpérieure perméable en ì la richesse de la végé. tation, on n'a cependam pan à redouter pour les golerien o'avancemem, l'envalissemon d'eaux abondanter, Danrcles sallér, le dolcser sablon. neux ch offina un bon appit aux remblain?.

" noraged d'actio. Les ouvrooger d'are courantre serom construits solidernem - mair coonomiqnement; on supprimerad l'emploi dela pievre de taille, qui peur-être remplacé par la macomnerie de briquen asec montier de cimen-. Pour les pontrs, on emploiera les tablicra métalliguar, qui ne donnam par hieu à des pousséer borizontalers, permettem d'etablir-les forrationn? danx des condition économiquess. En géneral lex foniationi sorone établieves sure le rocher, qu'on reneontre presque ci la surface dis dol, sur toun bs 


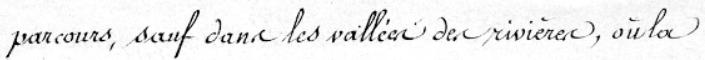
couche de sable arrive à de grander ćpaisseurse; Genrensemem le sable se prête à des combinaisonso de systemer de fondationso, à prixe de resiem non aléatove?

$$
\text { Les viaduar ne figurem par dann les }
$$

ousracger d'an prévure, si les trop grandere bauteurr de remblai, ne pawem être complitemem évitéro, dann les ravinn étroito en a pentes raidese, comme les trumchear adjacenter fournissem der Jéblain de rocher, on exicutero lex remblain ased des profith de valur, courber en redressen, on arrangera à la main, sourc forme de maconnerie à pierce sèche, len materiaux fournin par len trancheens, en encastram ha base du remblai danx le sol, pour en Diminuer l'empatimem. C'ess une solution employée ailleurs avec sucièn.

Statione.

$$
\text { La longueur totale au parcours ess infé- }
$$
rieure ò kil.en le nombre desstations presuers eshe Iln'y a donc par is préeccuper Der garer d'evitemem. Bouter les communer de la région trawersé, som desserviero. On donnera à la station on à la falte de chaque bealités tou le déseloppemem, toutes les constructionir? es engina utilese; comme une enquête spéciale reigles ler condition d'établissemem de stations, il u'y

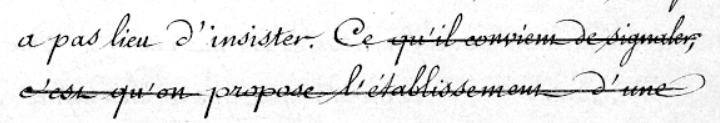


Station à Goulon, au poin de dépau de la ligne, kies au champ de Mbans, aux aborde du pon maxchand; une voie spéciale mettra en communi cation avec le chemin de for an littoral, la gare des vorkageurn en la gare der marchamdisen de la Compraynie de Parir is fyon ew àla Móditerranie. Ses voyageurs arrisam à Eoulon parfa grande ligne, trouverom donc, en gare, un train pour lero transporter-danc les station Ju littoral; inversemen, iloc serom ramenen aussi en gare. Ses habitantro de Foulon en de la kanlieues partiom danco las gare spéciale en s'y arrieterom au retour. Nooure n'avour par à insisker sur la convenonce quil ya, de créer à Eoulon f ville de 70.000 amere, poim commercial tries.impprtant, une gares spéciale qui aurain le grant avantage d'être tièr rapprocheé Zer quartiens les phwo densen a fer phure papuliso de cette grande villes. Gu poin d'arrivée de la ligne, la même combinaison esp adoptée. Ane soie spéciate meltra en communication directe les station de las grandeligne en bu chemin du littoral à ghe Raphael So Station de Tréjuro ess indiquee? prè du boulevaro neuf, qui conduin de laville à laf plage; on a infléchi le tracé pour diminuer la bistance de laville à lastation.

Superstructure enmateriel Tl n'yarien de particulier à dive suroulam. Ler bätimentr ger stations, sur les hablese es 
Bases d'estimation quais, sur be materiel roulam. Oéjä der typero spéciaux, on, non senlemem l'approbation De l'administration supérieure, mais la sanction de liexpérience, au poim de vue, de l'économie J'éta. blissement ez au bon fonctionnemen. Courclere perfectionnemento serom min à profic danno la construction der engind de traction en $\partial_{e}$ tronspore. La facilité au passage Danales courbes, surles penter en sur leno rampers, sera necherchée avec soin; la sécurité en l'économe, ganro l'entritien et le renouvellemem du matériel serom ler conséquencer de la réalisation de ce programme. des dépensere.

Ses travaux onn été estimèr aussiexactemem en aussi modérémem que possible, en prenam pour baser d'estimation, lese prix J'exécution de travaux analogueno, faits dann la région au midi. On a deja dir que ler fordatiour Jer ouvragen ne Jonnatimk par lieu às der aliano. Les router en construction on construi tero, paralliter à la côte gênem prarfoirc l'établissemen au chemin de fer; mairo ellen hiv viendrom aussi en aide, parla phur grande facilité des transpotiso der approsiosionnements. Les pria presur pour les souterrainn som faiblese, cerc prix s'appliquent cependam à der travaux en turmel présentam der conditions d'exécution analoguero. Ses prix applinguen anx ponceaux es aux ponto sons aussi peuélevéro, en tenant 
10

compte yu'ils s'appliquem à l'ouserture deres ousrager en non à leur longueur totale. (a) espère, parlex étuder Definitiver pousoir Diminuer la longueur, trons versale ì l'cuce du chemins, de quelques ponceaux sour remblai; on pense aussi, que le sable, qu' on trouve danx leve salliexs, permen lidoption de fondations moins couteusen que len vaser mollers, qui w'om pano été signaléers; les tabliers métalliquer permetten, De leur cité, Der économier dancles fondations des culéen de pontro.

Grafic probableo.

Des étuder sérieuser Du trafic probables

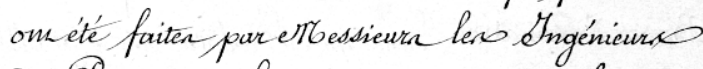
Des Sontro en Chausséer, äproporo auchemin de fer du littoual, projeté entre Hyeren es Tréjuns. Cers étudero on été soumisen au Conseil Général, Dane un eRappor de eri" Dyrion, Ingémeur Des Pontroen Ofausseén, en date duig Mbarse 1882, appronwé par e $16^{\circ}$. L' Ingénieur en chef le 11 Coxil suivam; eller conchorien, ähe suite?

* de liapplication des deux méthoden, de Nti: F. Mbichel, en du comptage des colliero, à une recette brute? hilométrique probuble de Ggso frunero.

$$
\text { e Nous pensone que ce chiffre peur étres }
$$
conservé, comme minimum, pour recelte probable? Delaligne entrères, de Erulon à Srejus st Ruphaiel, parce qu'ily aura entre Eoulon en Hyeres un dervice de bantieue trino rémunémateur. Les garess 
du Prader, de Carqueyrane en d'c Almanare, avec cellex de Eoulon a d'Thyereso, construites sur la ligne projetée, offrem en effer aux populations, agglomééen sur cette partie dn trace', Den facilités de transport qui seronn mises à profir dan une large mesure.

Coum aux froin d'exploitation d'une ligne à voie étroite, $l$ 'expérience a conduin $l^{\prime}$ 'administration supérieure, a adopter pour lewr calcul, des formulens peu difforenter les uner des autrer; celle qui semble le mienxe convenir a la ligne projetée, on raison des fortes ramper de son profil en long, ess la suivante: $2000 \stackrel{f}{f} \frac{4}{10}$ (e recelte brute).

$$
\text { (Empôts déduita) }
$$

sans qu'on ancun cas, l'application de cette formules aim pour offer, de faire descendre les facia, au-dessour On minimum de thoor "f par Tilometre.

Condusionso. ¿'Pesumone, dans ses grandes ligner, l'en. semble de l'asan-projet, présenté pow l'éablissement du Chemin de for du littoral, entre Eoulon en. Tréjuen.

Sth-e Raphael. Pix du Kilometre y compria 8.500:" du moteriel roulant 80 K Pein total d'etoblifoment 126. 000

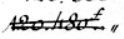
Lax total atabligement- "

Recette kilométrique probable de début $6.980^{f} . "$

Frain J'exploitation correspondant $4.79^{2.11}$ Orntérétr, par an, Dn (apital engagé $5 \%$ 
CAinsi dann l'boypothèse du ebiffre de trafic avrété

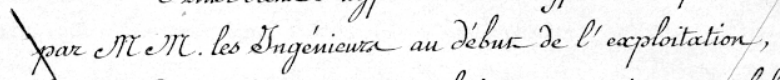
le service des intérétap on capital de construction, par kilo-

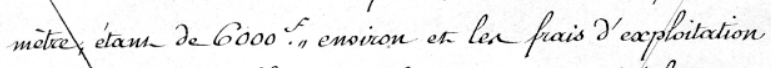
$479^{2}$ Xoin ensemble $1079^{2}{ }^{f}$; J'un antre coté, la recelte brute étank de $6 g 80$ france, il y awrait une mouffisance de 3812 "A par kilometre en de 379675 " four la ligne entierse. Le dexnandeur en concession propose que le $\mathscr{D}_{e^{\prime}}$ partement venille bien s'engager at convriz celte insuf. fisance, tam à l'aide de ses propress ressourcess qu'an moyen de la participation de f'Gtat, des Communes ot autrer intéressén, suivart compention à intersenic.

Quelques années onffircient pour faire Dispanâ.

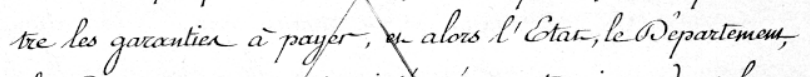
les Communer en autres interessén, entreraiem Jans le partage des excédante de recettesd, suivam laproportion des avancer foites en jusqu'à concurrence du rembourse. menk complert de cer avancer, sand intéréta:

La proportion oroissante des recettes est certaine, el ler sacrificer pécuniciores demandés, qui n'aurom qu'une Durée fimité, som amplememn justifió par tour les asantager qui résulteront pour le Oépartemen 2k Sar de la

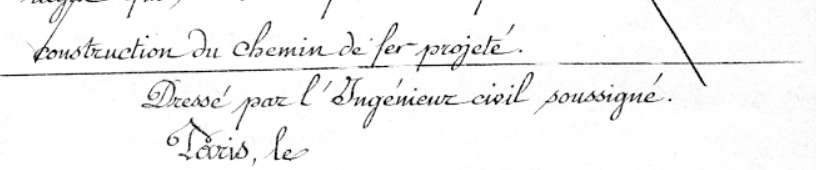

